\title{
WCC 2017-A-104: ANALYSIS OF HEART AND LUNG DOSES IN LEFT BREAST CANCER PATIENTS TREATED WITH RADIATION
}

\author{
Kishore Kumar Kota, Monica Malik Irukulla, Syed Fayaz Ahmed, Deepa M Joseph
}

\section{BACKGROUND:}

Radiotherapy forms a part in the multimodality treatment approach in breast cancers. Radiation reduces the risk of cancer recurrence and death. However, it usually involves some radiation exposure to the underlying heart and lungs. More challenging is the management of left sided breast cancers where unintended dose to heart is of high cocern. Our aim was to analyze the radiation doses to heart and lungs.

\section{MATERIALS AND METHODS:}

A retrospective analysis was done in patients of left breast cancer, admitted for radiotherapy from Jan 2013 to December2016. A total of 65 patients were included for analysis. All patients were simulated after making thermo plastic mask for immobilization. All patients were treated with EBRT using either hypo fractionated or conventional fractionation by 3DCRT technique. Heart and lung doses were recorded from treatment planning system.

\section{RESULTS:}

Median age was 53years. Out of these $15(23 \%)$, patients had diabetes, $23(33 \%)$ patients were hypertensive, 6 (10\%) patients had preexisting heart disease, and $3(5 \%)$ patients had hypothyroidism. Most of the patients were postmenopausal $(69 \%)$ followed by premenopausal $(15 \%)$ and perimenopausal $(10 \%)$. Most common site of the lump was upper outer

Article received on 25 FEB 2017, published on 08 MAR 2017.

Kishore Kumar Kota ${ }^{1}$, Monica Malik Irukulla ${ }^{2}$,Syed Fayaz Ahmed ${ }^{3}$,Deepa M Joseph $^{4}$

${ }^{1}$ Junior Resident, Department of Radiation Oncology,NIMS,India

${ }^{2}$ Associate Professor \&I/C HOD, Department of Radiation Oncology, NIMS, India

${ }^{3}$ Assistant Professor,Department of Radiation Oncology, NIMS, India

${ }^{4}$ Assistant Professor,Department of Radiation Oncology, NIMS, India

Corresponding Author:Kishore Kumar Kota

Email: kishorekumarmedico@gmail.com quadrant $(36 \%)$ followed by upper inner quadrant $(20 \%)$, central $(12.3 \%)$, lower inner $(7.6 \%)$ and lower outer quadrant (4.6\%). 45 patients underwent modified radical mastectomy

(MRM), 13 underwent breast conservative surgery (BCS), 4 patients underwent BCS followed by MRM, and no surgery was done in one patient. $42(64 \%)$ cases

Were hormone receptors positive. 19(29\%) patients were HER-2nue positive, 37 were negative and equivocal results were found in 8 patients. 58 patients received anthracycline based chemotherapy (52 adjuvant and 6 neoadjuvant) whereas 3 patients received nonanthracycline based chemotherapy. Out of 19 HER2 nue positive patients, 6 patients received Trastuzumab. Mean heart doses ranged between 1.4Gy to 23.3Gy with an average of $8.3 \mathrm{~Gy}$. V15 for Left Lung was found to be between $15.6 \%$ to $48.5 \%$ with an average of $34.6 \%$ for the patients who were treated with hypo fractionation. V20 for Left Lung was found to be between $27.9 \%$ to $56.3 \%$ with an average of $38.1 \%$ for the patients who were treated with conventional fractionation. Ten patients died during follow up, of which one was due to cardiac cause (AWMI), other deaths were related to distant metastasis. 4 year overall survival was $80 \%$.

\section{CONCLUSION:}

Radiation therapy given to left breast cancer patients can potentially lead to late effects on heart and lungs. Anthracycline based chemotherapy and trastuzumab can also lead to cardiac morbidity patients need to be monitored during follow up for late effects. Our early results did not show any significant cardio pulmonary morbidity. 